\title{
Detectability of inflationary gravitational waves with microwave background polarization
}

\author{
Marc Kamionkowski* \\ Department of Physics, Columbia University, 538 West 120th Street, New York, New York 10027 \\ Arthur Kosowsky ${ }^{\dagger}$ \\ Harvard-Smithsonian Center for Astrophysics, 60 Garden Street, Cambridge, Massachusetts 02138 \\ and Department of Physics, Lyman Laboratory, Harvard University, Cambridge, Massachusetts 02138
}

(Received 28 May 1997; published 26 November 1997)

\begin{abstract}
Inflation predicts specific relations between the amplitudes and spectral indices of the primordial spectrum of density (scalar metric) perturbations and gravitational waves (tensor metric perturbations). Detection of a stochastic gravitational-wave background is essential for identifying this unmistakable signature. Polarization of the cosmic microwave background can isolate these tensor modes in a model-independent way and thereby circumvent the cosmic-variance limit to detecting a small tensor signal with only a temperature map. Here we assess the detectability of a gravity-wave background with a temperature and polarization map. For detector sensitivities better than $10-20 \mu \mathrm{K} \sqrt{\mathrm{sec}}$, the sensitivity to a tensor signal is always dominated by the polarization map. With a detector sensitivity of order $1 \mu \mathrm{K} \sqrt{\mathrm{sec}}$, polarization could improve on a temperature-map sensitivity to tensor modes by two to three orders of magnitude. Even a small amount of reionization substantially enhances tensor-mode detectability. We also argue that the sensitivity of the Planck Surveyor to tensor modes is significantly improved with polarization, even taking into account the resulting degradation of the intensity determination in the high-frequency channels. [S0556-2821(98)01102-3]
\end{abstract}

PACS number(s): 98.70.Vc, 98.80.Cq

\section{INTRODUCTION}

Slow-roll inflation provides a unified and testable paradigm for understanding the flatness and smoothness of the Universe and the origin of structure. The predictions of a flat Universe [1,2] and a nearly scale-invariant spectrum of primordial density perturbations (scalar metric perturbations) [3] will be tested with unprecedented precision by forthcoming cosmic microwave background (CMB) temperature maps [4-6] from experiments such as Microwave Anistrophy Probe (MAP) [7] and the Planck Surveyor [8]. Inflation also predicts a nearly scale-invariant stochastic gravitationalwave background (tensor metric perturbations) [9]. A flat Universe with nearly scale-invariant adiabatic scalar and tensor perturbations would certainly suggest that inflation occurred but would not provide incontrovertible evidence. However, specific relations between the "inflationary observables,' the amplitudes and spectral indices of the scalar and tensor power spectra, are unique predictions of inflation [10]. Verification of these relations would provide a "smoking-gun", signature of inflation. For this reason, detection of the gravitational-wave background is of the utmost importance for an unambiguous test of inflation.

Scalar and tensor metric perturbations both contribute to temperature and polarization fluctuations in the CMB. A temperature map, even if ideal, will never determine the tensor amplitude with a standard error better than around $30 \%$ of the total perturbation amplitude: cosmic variance from the dominant scalar perturbations provides a fundamental limit to the sensitivity of $\mathrm{CMB}$ temperature maps to tensor pertur-

\footnotetext{
*Email address: kamion@phys.columbia.edu

†Email address: akosowsky@cfa.harvard.edu
}

bations (the cosmic variance in a signal is proportional to the total signal). However, the scalar and tensor contributions to CMB polarization can be geometrically decomposed in a model-independent fashion, so one can circumvent the cosmic-variance limit present in temperature maps $[11,12]$. The polarization tensor $P_{a b}(\hat{\mathbf{n}})$ on the celestial sphere can be decomposed into its "gradient" (or "curl-free") and "curl", parts, in analogy to the decomposition of a vector field. Scalar perturbations have no handedness, so they cannot give rise to a curl component. On the other hand, tensor perturbations do have a handedness, so they induce a curl component. The cross-correlation between temperature and polarization can also help isolate the tensor contribution, although this signature may have some model dependence [13].

In this paper, we assess the detectability of inflationary tensor modes given a full-sky polarization and/or temperature map with a given angular resolution and level of instrumental noise. In other words, for what tensor amplitudes can one hope to make an unambiguous statistically significant detection of tensor modes with a given experiment? We answer this question in two ways: First, we determine the minimum tensor amplitude which would be observationally accessible with only the curl component in the polarization field as a function of detector sensitivity. This will provide a conservative, yet model-independent, estimate for the sensitivity. We then consider the tensor amplitude which would be detectable by fitting all the temperature and polarization auto- and cross-correlation functions to inflationary predictions, and translate the results into implications for specific models of inflation. Previous work has addressed the related question of how precisely MAP and Planck can determine the tensor amplitude assuming a model with a given (somewhat optimistic) tensor amplitude $[12,14]$.

For experiments with detector sensitivities near 
$10 \mu \mathrm{K} \sqrt{\mathrm{sec}}$, the tensor-mode detectability with a polarization map can improve on that from a temperature map alone by an order of magnitude. Even with detectors substantially less sensitive, polarization can provide large improvements in tensor detectability for cases where the temperature map is not very restrictive, as when fitting many cosmological parameters to a single data set. In this case, the temperaturepolarization cross-correlation, which has a larger amplitude than the curl polarization component, becomes important. Detector sensitivities attainable in the foreseeable futuresay $1 \mu \mathrm{K} \sqrt{\mathrm{sec}}$-will dramatically enhance our ability to see tensor perturbations; tensor-scalar ratios as small as $10^{-4}$ might be probed. Even a small amount of reionization significantly improves the detectability of tensor modes. Needless to say, these experimental requirements pose great challenges, and errors in such measurements may well be dominated by systematic effects such as polarized foreground emission. However, the cosmological significance of these small signals will hopefully spur continued experimental improvements and further efforts to understand and isolate foregrounds.

Below, we begin with a review of the inflationary observables in Sec. II. Section III reviews the gradient or curl decomposition and the temperature-polarization power spectra. In Sec. IV, we calculate the model-independent tensor sensitivity achievable with measurements of only the curl component of the polarization. We then consider what can be learned from only a temperature map and from a combined temperature-polarization map. Section V compares these sensitivities with the predictions of some specific inflationary models, and some concluding remarks follow in Sec. VI.

\section{THE INFLATIONARY OBSERVABLES}

Inflation occurs when some scalar field $\phi$ (the "inflaton") is displaced from the minimum of its potential $V(\phi)$ such that the energy density of the Universe is dominated by the field's potential energy for a time long compared with the Hubble time. During this inflationary phase, the expansion of the Universe accelerates, small quantum fluctuations in $\phi$ produce classical scalar perturbations, and quantum fluctuations in the spacetime metric produce gravitational waves. If the inflaton potential $V(\phi)$ is given in units of $m_{\mathrm{Pl}}^{4}$, and the inflaton $\phi$ is in units of $m_{\mathrm{Pl}}$, then the scalar and tensor spectral indices are [15]

$$
\begin{gathered}
1-n_{s}=\frac{1}{8 \pi}\left(\frac{V^{\prime}}{V}\right)^{2}-\frac{1}{4 \pi}\left(\frac{V^{\prime}}{V}\right)^{\prime}, \\
n_{t}=-\frac{1}{8 \pi}\left(\frac{V^{\prime}}{V}\right)^{2} .
\end{gathered}
$$

The amplitudes can be fixed by their contribution to $C_{2}^{\mathrm{TT}}$, the quadrupole moment of the CMB temperature,

$$
\begin{gathered}
\mathcal{S} \equiv 6 C_{2}^{\mathrm{TT}, \text { scalar }}=33.2\left[V^{3} /\left(V^{\prime}\right)^{2}\right], \\
\mathcal{T} \equiv 6 C_{2}^{\mathrm{TT}, \text { tensor }}=9.2 V .
\end{gathered}
$$

The scalar amplitude may be up to $\sim 20 \%$ larger for plausible values of the cosmological constant; for numerical corrections, see $[17,16]$. For the slow-roll conditions to be satisfied, we must have

$$
\begin{gathered}
(1 / 16 \pi)\left(V^{\prime} / V\right)^{2} \ll 1, \\
(1 / 8 \pi)\left(V^{\prime \prime} / V\right) \ll 1,
\end{gathered}
$$

which guarantee that inflation lasts long enough to make the Universe flat and to solve the horizon problem.

When combined with Cosmic Background Explorer (COBE) results, current degree-scale anisotropy and largescale structure observations suggest that $\mathcal{T S}$ is less than order unity in inflationary models, which restricts $V \leqq 5 \times 10^{-12}$. If the consistency relation $\mathcal{T} \mathcal{S} \simeq-7 n_{t}[\mathrm{im}$ plied by Eqs. (1) and (2)] holds, the tensor spectrum must be nearly scale invariant $\left(n_{t} \simeq 0\right)$.

\section{STATISTICS OF CMB ANISOTROPIES AND POLARIZATION}

\section{A. Harmonic expansion}

A temperature map $T(\hat{\mathbf{n}})$ of the sky can be expanded in spherical harmonics,

$$
\frac{T(\hat{\mathbf{n}})}{T_{0}}=1+\sum_{l m} a_{(l m)}^{\mathrm{T}} Y_{(l m)}(\hat{\mathbf{n}}),
$$

where the mode amplitudes are given by

$$
a_{(l m)}^{\mathrm{T}}=\frac{1}{T_{0}} \int d \hat{\mathbf{n}} T(\hat{\mathbf{n}}) Y_{(l m)}^{*}(\hat{\mathbf{n}}) ;
$$

this follows from the orthonormality of the spherical harmonics.

The Stokes parameters $Q(\hat{\mathbf{n}})$ and $U(\hat{\mathbf{n}})$ (where $Q$ and $U$ are measured with respect to the polar $\hat{\theta}$ and azimuthal $\hat{\phi}$ axes) which specify the linear polarization in direction $\mathbf{n}$ are components of a $2 \times 2$ symmetric trace-free $(\mathrm{STF})$ tensor,

$$
\mathcal{P}_{a b}(\hat{\mathbf{n}})=\frac{1}{2}\left(\begin{array}{cc}
Q(\hat{\mathbf{n}}) & -U(\hat{\mathbf{n}}) \sin \theta \\
-U(\hat{\mathbf{n}}) \sin \theta & -Q(\hat{\mathbf{n}}) \sin ^{2} \theta
\end{array}\right),
$$

where the subscripts $a b$ are tensor indices. Just as the temperature is expanded in terms of spherical harmonics, the polarization tensor can be expanded [18]

$$
\frac{\mathcal{P}_{a b}(\hat{\mathbf{n}})}{T_{0}}=\sum_{l m}\left[a_{(l m)}^{\mathrm{G}} Y_{(l m) a b}^{\mathrm{G}}(\hat{\mathbf{n}})+a_{(l m)}^{\mathrm{C}} Y_{(l m) a b}^{\mathrm{C}}(\hat{\mathbf{n}})\right],
$$

in terms of the tensor spherical harmonics $Y_{(l m) a b}^{\mathrm{G}}$ and $Y_{(l m) a b}^{\mathrm{C}}$, which are a complete basis for the "gradient" (i.e., curl-free) and "curl" components of the tensor field, respectively. (See Ref. [19] for an alternative but equivalent formalism.) The mode amplitudes are given by

$$
a_{(l m)}^{\mathrm{G}}=\frac{1}{T_{0}} \int d \hat{\mathbf{n}} \mathcal{P}_{a b}(\hat{\mathbf{n}}) Y_{(l m)}^{\mathrm{G} a b *}(\hat{\mathbf{n}}),
$$




$$
a_{(l m)}^{\mathrm{C}}=\frac{1}{T_{0}} \int d \hat{\mathbf{n}} \mathcal{P}_{a b}(\hat{\mathbf{n}}) Y_{(l m)}^{\mathrm{C} a b *}(\hat{\mathbf{n}}),
$$

which can be derived from the orthonormality properties,

$$
\begin{gathered}
\int d \hat{\mathbf{n}} Y_{(l m) a b}^{\mathrm{G} *}(\hat{\mathbf{n}}) Y_{\left(l^{\prime} m^{\prime}\right)}^{\mathrm{G} a b}(\hat{\mathbf{n}})=\delta_{l l^{\prime}} \delta_{m m^{\prime}}, \\
\int d \hat{\mathbf{n}} Y_{(l m) a b}^{\mathrm{C} *}(\hat{\mathbf{n}}) Y_{\left(l^{\prime} m^{\prime}\right)}^{\mathrm{C} a b}(\hat{\mathbf{n}})=\delta_{l l^{\prime}} \delta_{m m^{\prime}}, \\
\int d \hat{\mathbf{n}} Y_{(l m) a b}^{\mathrm{G} *}(\hat{\mathbf{n}}) Y_{\left(l^{\prime} m^{\prime}\right)}^{\mathrm{C} a b}(\hat{\mathbf{n}})=0 .
\end{gathered}
$$

Here $T_{0}$ is the cosmological mean CMB temperature and we are assuming $Q$ and $U$ are measured in brightness temperature units rather than flux units. Scalar perturbations have no handedness. Therefore, they can produce no curl, so $a_{(l m)}^{\mathrm{C}}=0$ for scalar modes. On the other hand tensor modes do have a handedness, so they produce a nonzero curl, $a_{(l m)}^{\mathrm{C}} \neq 0$.

\section{B. Statistics}

A given inflationary model predicts that the $a_{(l m)}^{\mathrm{X}}$ are Gaussian random variables with covariance $\left\langle\left(a_{\left(l^{\prime} m^{\prime}\right)}^{\mathrm{X}^{\prime}}\right) * a_{(l m)}^{\mathrm{X}}\right\rangle=C_{l}^{\mathrm{XX}^{\prime}} \delta_{l l^{\prime}} \delta_{m m^{\prime}} \quad$ (for $\left.\quad \mathrm{X}, \mathrm{X}^{\prime}=\{T, G, C\}\right)$. Parity demands that $C_{l}^{\mathrm{TC}}=C_{l}^{\mathrm{GC}}=0$. Therefore the statistics of the CMB temperature-polarization map are completely specified by the four sets of moments, $C_{l}^{\mathrm{TT}}, C_{l}^{\mathrm{TG}}, C_{l}^{\mathrm{GG}}$, and $C_{l}^{\mathrm{CC}}$. Also, as stated above, only tensor modes will produce nonzero $C_{l}^{\mathrm{CC}}$

To illustrate, Fig. 1 shows the four temperaturepolarization power spectra. The dotted curves correspond to a COBE-normalized inflationary model with cold dark matter and no cosmological constant $(\Lambda=0)$, Hubble constant (in units of $100 h \mathrm{~km} \mathrm{sec}^{-1} \mathrm{Mpc}^{-1}$ ) $h=0.65$, baryon density $\Omega_{b} h^{2}=0.024$, scalar spectral index $n_{s}=1$, no reionization, and no gravitational waves. The solid curves show the spectra for a COBE-normalized stochastic gravity-wave background with a flat scale-invariant spectrum $(h=0.65$, $\Omega_{b} h^{2}=0.024$, and $\Lambda=0$ ) in a critical-density Universe. Note that the panel for $C_{l}^{\mathrm{CC}}$ contains no dotted curve since scalar perturbations produce no $\mathrm{C}$ polarization component. The dashed curve in the $\mathrm{CC}$ panel shows the tensor spectrum for a reionized model with optical depth $\tau=0.1$ to the surface of last scatter. We use the code CMBFAST [19] to generate all power spectra used in this paper (but note that the normalizations of the polarization $C_{l}$ 's from this code are different than those used in this paper [18]).

\section{Cosmic and pixel-noise variance}

Theory predicts the covariances $C_{l}^{\mathrm{XX}}$ of the distributions for the multipole coefficients $a_{(l m)}^{\mathrm{X}}$. For a given $l$, only $2 l+1$ independent harmonics $(m=-l, \ldots, l)$ are available to estimate these variances, giving a statistical "cosmic variance" limit to how well we can estimate each moment $C_{l}^{\mathrm{XX}}$ from a map. Instrumental noise, beam size, and sky coverage
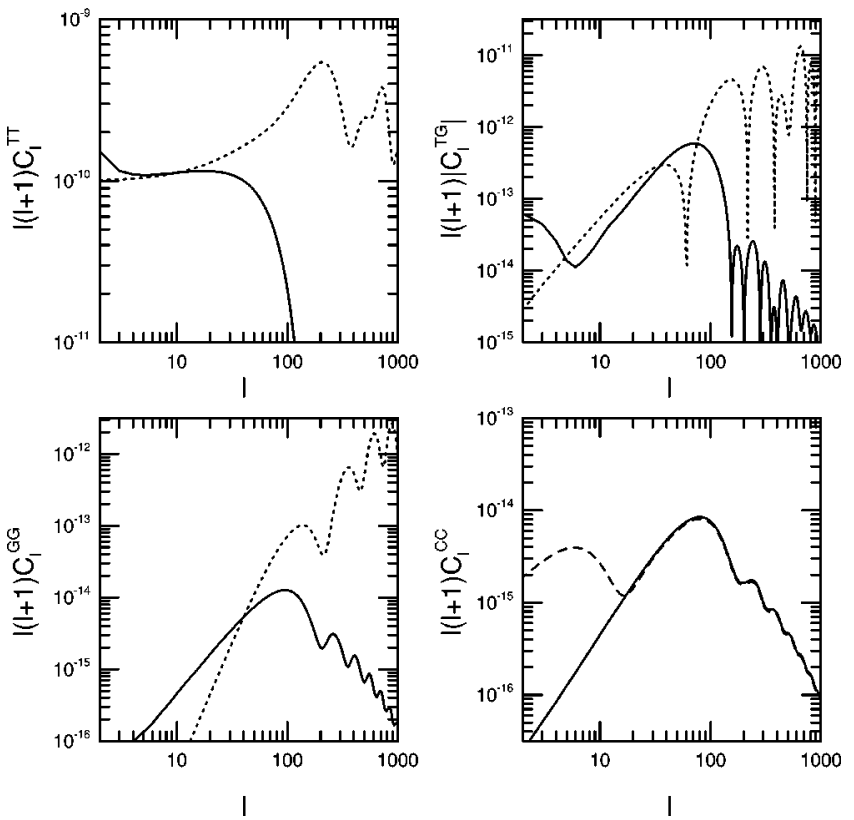

FIG. 1. Theoretical predictions for the four nonzero CMB temperature-polarization spectra as a function of multipole moment $l$. The solid curves are the predictions for a COBE-normalized inflationary model with no reionization and no gravitational waves for $h=0.65, \Omega_{b} h^{2}=0.024$, and $\Lambda=0$. The dotted curves are the predictions which would be obtained if the COBE anisotropy were due entirely to a stochastic gravity-wave background with a flat scaleinvariant spectrum (with the same cosmological parameters). Note that the panel for $C_{l}^{\mathrm{CC}}$ contains no dotted curve since scalar perturbations produce no " $C$ ", polarization component; instead, the dashed line in the lower right panel shows a reionized model with optical depth $\tau=0.1$ to the surface of last scatter.

also contribute to the variance in each moment. If $\widehat{C_{l}^{\mathrm{XX}}}$ is an estimator for the moment $C_{l}^{\mathrm{XX}}$, then the $(6 \times 6)$ covariance matrix for these estimators is

$$
\Xi_{\mathrm{AB}} \equiv\left\langle\left(\widehat{C_{l}^{\mathrm{A}}}-C_{l}^{\mathrm{A}}\right)\left(\widehat{C_{l}^{\mathrm{B}}}-C_{l}^{\mathrm{B}}\right)\right\rangle,
$$

for $\mathrm{A}, \mathrm{B}=\mathrm{XX}^{\prime}$. Expressions for the entries of this matrix for an idealized mapping experiment with a given instrumental noise, beamwidth, and fraction of sky covered are presented in Sec. III of Ref. [18].

\section{DETECTABILITY OF TENSOR MODES}

\section{A. Curl component of the polarization}

The curl component of polarization, $C_{l}^{\mathrm{CC}}$, provides a model-independent probe of tensor perturbations in inflationary models. What detector sensitivity is required to distinguish this signal from a null result? The answer to this question will of course depend on the angular spectrum of the curl component, or equivalently, the $l$ dependence of $C_{l}^{\mathrm{CC}}$. However, as noted above, current measurements indicate that $n_{t}$ must be close to zero if the inflationary relation $\mathcal{T} \mathcal{S} \simeq-7 n_{t}$ is satisfied. Therefore, in the following analysis we consider a scale-invariant $\left(n_{t}=0\right)$ tensor perturbation spectrum. Furthermore, variations in the other cosmological parameters have a relatively weak effect on the spectrum of 
the curl component (except for reionization, the effect of which is mentioned below), and it is likely these parameters will be fairly well determined by temperature maps and other observations.

Consider a mapping experiment which measures the temperature and polarization on the entire sky with beamwidth $\theta_{\mathrm{FWHM}}$ and a temperature sensitivity $s$ (which has units $\mu \mathrm{K} \sqrt{\mathrm{sec}})$, giving $N_{\text {pix }} \simeq 42,000\left(\theta_{\mathrm{FWHM}} / \mathrm{deg}\right)^{-2}$ independent pixels on the sky. The Gaussian beamwidth is $\sigma_{b} \equiv 7.42 \times 10^{-3}\left(\theta_{\mathrm{FWHM}} / 1^{\circ}\right)$. If the Universe has no tensor perturbations, then the $1 \sigma$ upper limit to the tensor amplitude in a null experiment is $\sigma_{\mathcal{T}}$, where

$$
\frac{1}{\sigma_{\mathcal{T}}^{2}}=\sum_{l}\left(\frac{\partial C_{l}^{\mathrm{CC}}}{\partial \mathcal{T}}\right)_{\mathcal{T}=0}^{2} \frac{1}{\left(\sigma_{l}^{\mathrm{CC}}\right)^{2}},
$$

and

$$
\sigma_{l}^{\mathrm{CC}}=\sqrt{2 /(2 l+1)} w^{-1} e^{l^{2} \sigma_{b}^{2}},
$$

is the pixel-noise variance with which $C_{l}^{\mathrm{CC}}$ can be determined (there is no cosmic variance if there is no cosmological signal). Here, $w^{-1}=4 \pi s^{2} /\left(t_{\text {pix }} N_{\text {pix }} T_{0}^{2}\right)$ is the inverse weight per unit area on the sky where $t_{\text {pix }}$ is the time spent observing each pixel, so $w^{-1}=2.14 \times 10^{-15} t_{\mathrm{yr}}^{-1}(s / 200$ $\mu \mathrm{K} \sqrt{\mathrm{sec}})^{2}$ with $t_{\mathrm{yr}}$ the total observing time in years. Since $\mathcal{T}$ is the overall normalization of the tensor spectrum, we can write $\partial C_{l}^{\mathrm{CC}} / \partial \mathcal{T}=C_{l}^{\mathrm{CC}} / \mathcal{T}$. Substituting the $C_{l}^{\mathrm{CC}}$ spectrum from Fig. 1 (no reionization) into Eq. (12) with $\theta_{\mathrm{FWHM}}=0.5$ gives

$$
\frac{\sigma_{\mathcal{T}}}{6 C_{2}^{\mathrm{TT}}} \simeq 5 \times 10^{-4}\left(\frac{s}{\mu \mathrm{K} \sqrt{\mathrm{sec}}}\right)^{2} t_{\mathrm{yr}}^{-1} .
$$

Thus, the curl component of a full-sky polarization map is sensitive to inflaton potentials $V \geqslant 5 \times 10^{-15} t_{\mathrm{yr}}^{-1}$ $(s / \mu \mathrm{K} \sqrt{\mathrm{sec}})^{2}$. Tensor modes produce polarization primarily on angular scales greater than a degree; the result in Eq. (14) will be similar for any $\theta_{\mathrm{FWHM}} \leq 1^{\circ}$. Improvement on current constraints with only the curl polarization component requires a detector sensitivity $s \lessgtr 40 t_{\mathrm{yr}}^{1 / 2} \mu \mathrm{K} \sqrt{\mathrm{sec}}$. Again, the curl component of polarization is due only to tensor perturbations and its shape is insensitive to the baryon density and Hubble constant.

Even a small amount of reionization will significantly increase the polarization signal at low $l$, as shown in the CC panel of Fig. 1, making the above result sensitive to the ionization history of the Universe. For example, in the same model with an optical depth to last scattering of $\tau=0.1$, the numerical factor in Eq. (14) becomes $9.3 \times 10^{-5}$, increasing sensitivity to the tensor modes by more than a factor of 5 . This level of reionization (if not more) is expected in cold dark matter models $[4,21,22]$, so Eq. (14), for no reionization, provides a conservative estimate.

\section{B. Full polarization and temperature spectra}

Fitting an inflationary model to the entire set of temperature and polarization power spectra can improve tensor detectability, especially at comparatively low sensitivities.
Generalizing Eq. (12), if the tensor amplitude is the only relevant parameter, the $1 \sigma$ sensitivity to the tensor amplitude is given by

$$
\frac{1}{\sigma_{\mathcal{T}}^{2}}=\sum_{l} \sum_{\mathrm{XX}^{\prime}}\left(\frac{\partial C_{l}^{\mathrm{X}}}{\partial \mathcal{T}}\right)_{\mathcal{T}=0}\left[\Xi^{-1}\right]_{\mathrm{XX}}\left(\frac{\partial C_{l}^{\mathrm{X}^{\prime}}}{\partial \mathcal{T}}\right)_{\mathcal{T}=0},
$$

where the second sum is over $\mathrm{X}, \mathrm{X}^{\prime}=\{\mathrm{TT}, \mathrm{TG}, \mathrm{GG}, \mathrm{CC}\}$. However, the TT, TG, and GG power spectra also have a strong dependence on other cosmological parameters. Furthermore, some parameters, such as the scalar-mode normalization and power-law index, can only be determined with any precision from the CMB. Multiple parameters can be accounted for with the curvature matrix (also known as the Fisher information matrix)

$$
\alpha_{i j}=\sum_{l} \sum_{\mathrm{XX}^{\prime}} \frac{\partial C_{l}^{\mathrm{X}}\left(\mathbf{s}_{0}\right)}{\partial s_{i}}\left[\Xi^{-1}\right]_{\mathrm{XX}} \frac{\partial C_{l}^{\mathrm{X}^{\prime}}\left(\mathbf{s}_{0}\right)}{\partial s_{j}}+P_{i j},
$$

where $\mathbf{s}_{0}$ are the parameters of the putative underlying cosmological model. The matrix $P_{i j}$ takes into account gaussian priors for these parameters $\mathbf{s}_{0}$ [6]; it is the inverse of the covariance matrix for the parameters determined from other measurements or observations. The covariance matrix is the inverse of the curvature matrix, $\mathcal{C}=[\alpha]^{-1}$, and the standard error in the tensor amplitude, after marginalizing over all other undetermined parameters, is $\sigma_{T}=\sqrt{\mathcal{C}_{T T}}$. Inclusion of the prior matrix $P_{i j}$ guarantees that the other undetermined parameters will be marginalized over only for reasonable values of those parameters. If it is not included, the results for $\sigma_{\mathcal{T}}$ are overly conservative.

Note that when testing the null hypothesis of no tensor perturbations, the underlying model $\mathbf{s}_{0}$ has no tensor contribution, so the derivatives of $C_{l}^{\mathrm{CC}}$ in Eq. (16) with respect to all the parameters except $\mathcal{T}$ are zero. In this case, the CC contribution to the variance decouples from the rest of the power spectra and has no dependence on the other cosmological parameters. However, even in this case, the other three power spectra depend on all the cosmological parameters, so the full curvature matrix must be calculated when comparing to the complete set of temperature and polarization data.

Figures 2 and 3 show the results for $\sigma_{T}=\sqrt{\mathcal{C}_{T T}}$, obtained from Eq. (16), as a function of detector sensitivity $s$ for a full-sky, one-year mapping experiment. We have used a beamwidth $0.5^{\circ}$, but the result is essentially independent of beam size for any beam smaller than one degree. In both cases, the parameters of the putative underlying model are those used in Fig. 1, and we again consider the sensitivity to a tensor spectrum with $n_{t}=0$. In both figures, the solid curve shows the results which would be obtained by fitting all four sets of temperature and polarization moments; the dotted line shows results which would be obtained by fitting only the temperature moments, while the dashed line shows those obtained by fitting only the $\mathrm{CC}$ polarization.

In Fig. 2, we make the optimistic assumption that all cosmological parameters except the scalar normalization $\mathcal{S}$ and spectral index $n_{s}$ are known. Therefore, we diagonalize the $3 \times 3$ covariance matrix for $\mathcal{T}, \mathcal{S}$, and $n_{s}$, assuming no prior information about any of these quantities. Figure 3 is more 


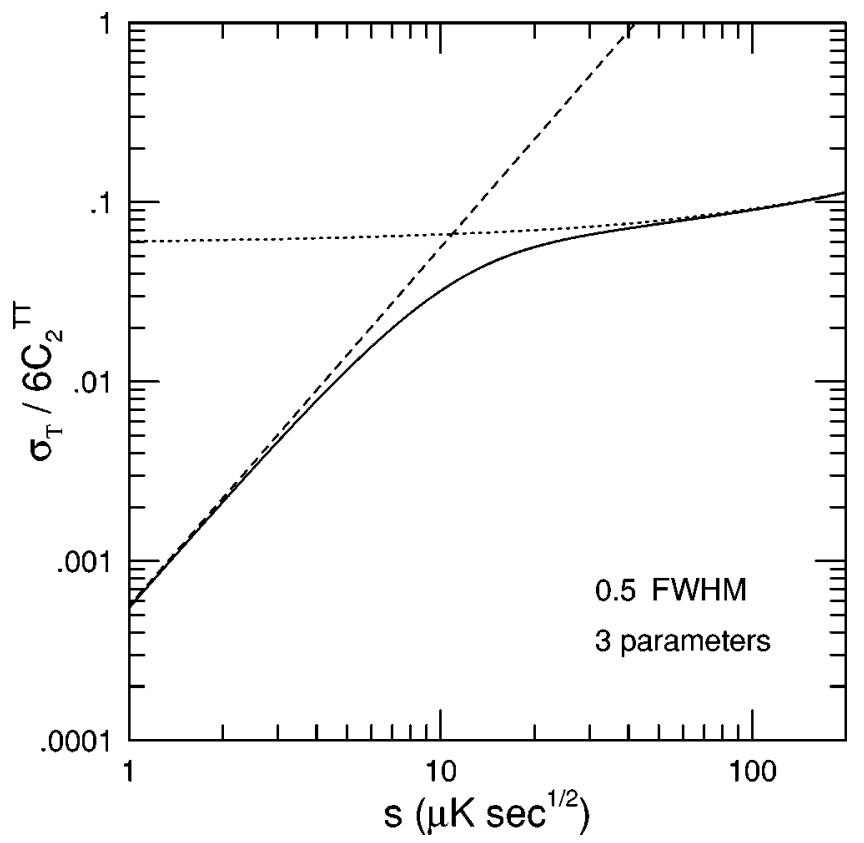

FIG. 2. Results for the $1 \sigma$ sensitivity $\sigma_{\mathcal{T}}$ to the amplitude $\mathcal{T}$ of a flat $\left(n_{t}=0\right)$ tensor spectrum as a function of detector sensitivity $s$ for an experiment which maps the CMB temperature and polarization on the full sky for one year with an angular resolution of $0.5^{\circ}$. The vertical axis is in units of the temperature quadrupole. Here, we have assumed that the spectra are fit only to $\mathcal{S}, \mathcal{T}$, and $n_{s}$, and the parameters of the cosmological model are those used in Fig. 1. The dotted curve shows the results obtained by fitting only the TT moments; the dashed curve shows results obtained by fitting only the $\mathrm{CC}$ moments; and the solid curve shows results obtained by fitting all four sets of moments.

conservative. Here we assume that $h, \Omega_{b} h^{2}, \Lambda$, the massiveneutrino density $\Omega_{\nu} h^{2}$, and $\tau$ are to be determined from the data as well as $\mathcal{S}, \mathcal{T}$, and $n_{s}$. We include only very conservative priors on these parameters: $1 \sigma$ Gaussian errors of 0.01 on $\Omega_{b} h^{2}, 0.3$ on $\Lambda, 0.15$ on $h, 0.5$ on $\tau$, and 0.2 on $\Omega_{\nu} h^{2}$.

For detectors sensitivities $s \geq 20 \mu \mathrm{K} \sqrt{\mathrm{sec}}$, the tensormode detectability with a three-parameter fit comes primarily from the temperature map, although polarization does provide some incremental improvement. However, for detector sensitivities $s \lesssim 10 \mu \mathrm{K} \sqrt{\mathrm{sec}}$, the sensitivity to tensor modes comes almost entirely from the polarization. For the eightparameter fit (which is probably closer to the types of fits which will be done with satellite data), the determination of the tensor amplitude is always dominated by polarization, although in this case the temperature-polarization crosscorrelation gives most of the contribution at larger values of $s$. Note that the solid curves (from fitting to the complete temperature and polarization power spectra) in Figs. 2 and 3 asymptote to the dotted curves (from fitting only to the CC spectrum) at smaller $s$. This indicates that for better detector sensitivities, the determination of the tensor amplitude comes primarily from the curl component of the polarization and thus is largely independent of the other cosmological parameters. Any beam size below about a degree will be small enough to detect virtually all of the $\mathrm{CC}$ signal. Beams smaller than this will only improve the sensitivity to tensor modes by better constraining the other cosmological parameters from the TT and TG spectra.

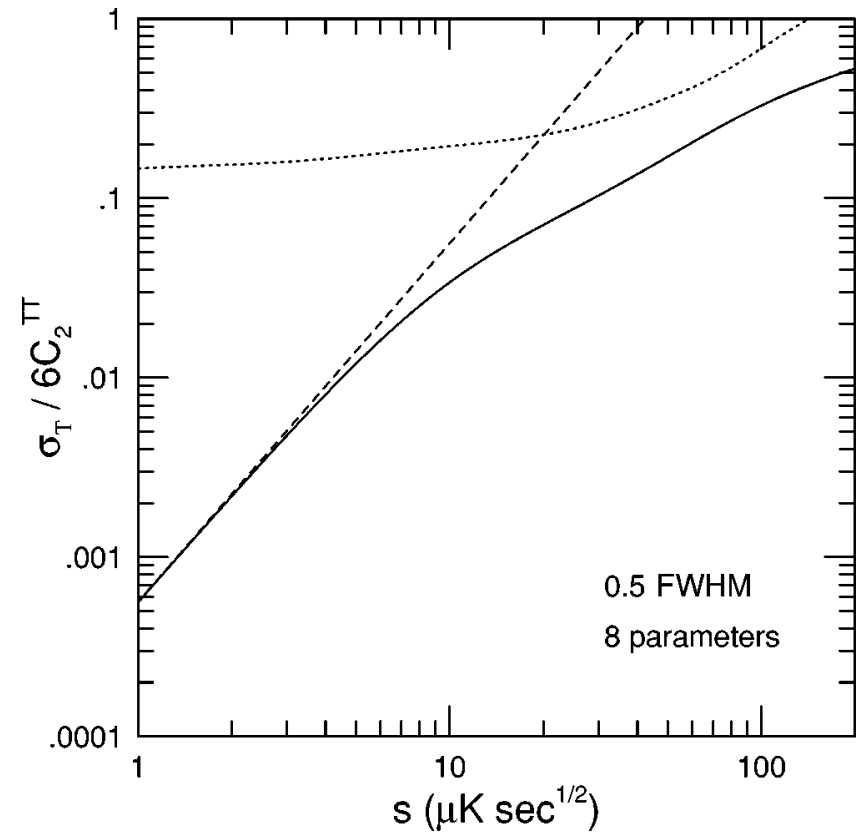

FIG. 3. The same as in Fig. 2, but here we assume that we will be fitting for $h, \Omega_{b} h^{2}, \Lambda, \tau$, and $\Omega_{\nu} h^{2}$ (with very conservative priors), in addition to $\mathcal{S}, \mathcal{T}$, and $n_{s}$.

In reionized models, the dashed CC curves in Figs. 2 and 3 move down substantially, while the dotted temperature curves remain essentially unchanged. As mentioned above, an optical depth of $\tau=0.1$ gives a factor of 5 improvement in detectability from the CC power spectrum, bringing $\sigma_{\mathcal{T}} / 6 C_{2}^{\mathrm{TT}}$ below $10^{-4}$ for the foreseeable detector sensitivity of $1 \mu \mathrm{K} \sqrt{\mathrm{sec}}$. Figures 2 and 3, for no reionization, are conservative estimates of tensor mode detectability.

With traditional bolometer detectors (e.g., the highfrequency channels on Planck), the polarization is determined by placing a polarizing filter in front of the detector, thereby halving the number of photons available for the temperature measurement. In this case, a temperature-only map with a given detector sensitivity $s$ should be compared with a polarized map of sensitivity $\sqrt{2} s$. Doing so, it is clear that for any detector sensitivity $s \lesssim 200 \mu \mathrm{K} \sqrt{\mathrm{sec}}$ (well above the sensitivity of the Planck high-frequency channels), the sensitivity to a tensor signal is improved significantly with the inclusion of polarization, even after taking into account the degradation of the temperature signal. Note that for Planck, an rms pixel noise of $\Delta T / T=2 \times 10^{-6}$ with angular resolution of $10^{\prime}$ [8] gives $s \simeq 25 \mu \mathrm{K} \sqrt{\mathrm{sec}}$. If the high-frequency channels are polarized, this number becomes $s \simeq 35 \mu \mathrm{K} \sqrt{\mathrm{sec}}$ after including the extra factor of $\sqrt{2}$.

\section{SOME SPECIFIC MODELS}

It is quite plausible that the amplitude of an inflationary stochastic gravity-wave background is large enough to be seen with a next-generation experiment. Slow-roll inflation provides a beautiful and economical explanation of isotropy, flatness, and the origin of density perturbations. However, we do not know the details of the physics responsible for inflation. Some models predict a sizeable tensor amplitude, while in others it is hopelessly small. Unfortunately, no con- 
sensus exists among theorists as to which are most likely. To illustrate the state of theoretical expectations for the amplitude of tensor modes, this Section give a brief overview of some inflationary models. Rather than survey the plethora of specific models individually, we follow the classification of Ref. [23]. We also suggest Ref. [24] for an intriguing parallel discussion.

In a large-field polynomial potential (e.g., chaotic inflation [25]), $V(\phi) \propto \phi^{p}$ with $p>1$, the expected tensor amplitude is $\mathcal{T} \simeq 1.4 \times 10^{-9} p /(p+200)$. This tensor amplitude will be detectable through the curl polarization component alone with a sensitivity $s \simeq 16 \mu \mathrm{K} \sqrt{\sec }$ for $p=2$, for example. Therefore, the tensor modes should be accessible with Planck if this is the correct model for inflation.

In small-field polynomial potentials, $V(\phi)$ $\propto\left[1-(\phi / \mu)^{p}\right]$ with $\phi \ll \mu \ll m_{P l}$ and $p>2$, the tensor spectrum is unobservably small. This is the type of potential expected if inflation occurs from a spontaneous-symmetrybreaking transition such as those envisioned in new inflation [2]. Similarly, in small-field quadratic potentials, $V(\phi)$ $\propto\left[1-(\phi / \mu)^{2}\right]$ with $\phi \ll \mu$, the tensor amplitude is unobservably small. Such a potential arises, e.g., in "natural inflation" models [26].

In a linear potential, $V(\phi) \propto \phi$, the tensor amplitude is proportional to the deviation of the scalar amplitude from unity; i.e., $\mathcal{T} \simeq 7 \times 10^{-10}\left(n_{s}-1\right)$. To detect such a signal through the curl polarization component alone requires a sensitivity $s \simeq 60 \mu \mathrm{K} \sqrt{\sec }\left[\left(n_{s}-1\right) / 0.3\right]^{1 / 2}$. A similar situation arises in exponential models, $V(\phi)$ $\propto \exp \sqrt{16 \pi \phi^{2} / p m_{P l}^{2}}$ with $p>0$. However, the constant of proportionality differs slightly. These models require a sensitivity $s \simeq 40 \mu \mathrm{K} \sqrt{\sec }\left[\left(n_{s}-1\right) / 0.3\right]^{1 / 2}$ to detect the tensors with only the curl polarization component. Therefore, if the scalar tensor index deviates by $\mathcal{O}(10 \%)$ - which is consistent, although not necessarily indicated, by COBE-this tensor signal can be detected with Planck. On the other hand, if $n_{s}$ is in fact very close to unity, the tensor signal might turn out to be unobservably small. In hybrid inflation models (those which require two fields for inflation), $V(\phi)$ $\propto\left[1+(\phi / \mu)^{p}\right]$ with $\phi<\mu$ and $p \geqslant 2$ [27], the tensor amplitude is only constrained to be smaller than that in exponential models, so a tensor signal may be observable in such models.

To summarize, some reasonable inflationary models give a tensor perturbation signal within reach of next-generation polarization experiments, whereas in many others the signal will be elusive. As this brief survey illustrates, CMB polarization can help discriminate between models. Detection of tensor modes would dramatically narrow the available range of models and determine the energy scale of inflation, while a null result would also provide interesting constraints on models.

\section{DISCUSSION AND CONCLUSIONS}

Detection of a stochastic gravity-wave background is essential to test the full predictions of slow-roll inflation. Here we have evaluated the detectability of tensor metric perturbations with a polarization map of the CMB. Inclusion of polarization will always improve on the tensor-mode sensitivity achievable with only a temperature map. For detector sensitivities $s \geq 10-20 \mu \mathrm{K} \sqrt{\mathrm{sec}}$, the improvement is incremental and comes primarily from the temperaturepolarization cross-correlation; for $s \lessgtr 10-20 \mu \mathrm{K} \sqrt{\mathrm{sec}}$, the improvement is dramatic and tensor-mode detectability is dominated by the polarization, particularly the curl (CC) component of the polarization. For detector sensitivities of $s=1 \mu \mathrm{K} \sqrt{\mathrm{sec}}$ the improvement is by two to three orders of magnitude. Polarization will significantly enhance the sensitivity to tensor modes for the high-frequency channels on Planck, even though the temperature measurement must be degraded to accommodate polarization. The tensor signature in the TT, TG, and GG spectra is somewhat model dependent, but the CC spectrum, which dominates the tensor signal with better detector sensitivities, provides an unambiguous model-independent probe of the stochastic gravity-wave background.

The ability of a temperature map to detect tensor perturbations is limited by cosmic variance to around $30 \%$ of the total perturbation amplitude. Some prior work $[28,29]$ concluded that cosmic variance would also limit the sensitivity of a polarization map to tensor modes. However, these papers did not take into account the geometric decomposition of scalar and tensor modes which is possible with a polarization map. Since scalar perturbations make no contribution to the CC polarization spectrum, the cosmic-variance limitations are effectively circumvented. The detectability of tensor modes with terrestrial and space-based gravitationalwave detectors was addressed in Ref. [29], although we believe that $\mathrm{CMB}$ polarization provides a more promising avenue toward detection of the predominantly longerwavelength inflationary gravitational radiation.

We have also reviewed some inflationary models. Predictions for the tensor amplitude differ greatly among plausible models; a high-sensitivity polarization map will help discriminate between inflationary models. In the event of a positive detection, the relations between the inflationary observables can be tested: the relation $\mathcal{T S} \simeq-7 n_{t}[10]$ must be satisfied. Several authors have previously investigated how precisely the inflationary observables can be determined with a temperature map alone $[5,6,23,16]$ and with a temperaturepolarization map [14] for some assumed (perhaps optimistic) models in which the amplitude of the tensor signal is large enough to be detected. While cosmic variance from scalar modes essentially precludes a temperature map from determining $n_{t}$, the CC polarization spectrum isolates the gravitational waves and thereby allows determination of $n_{t}$. For example, with $\mathcal{T} \mathcal{S}=0.05$ and a detector sensitivity of a few $\mu \mathrm{K} \sqrt{\mathrm{sec}}, n_{t}$ may be determined with a standard error of 0.05 or better by a one-year mapping experiment.

We note briefly that polarization measurements at very high sensitivities will require projecting out contributions from foreground polarized emission. While the characteristics and amplitudes of polarization foregrounds are unknown at present, the valuable cosmological signals contained in CMB polarization warrant intensive study of this question. We also note that the best current detectors are nearing photon shot-noise limits; the best Planck bolometers, at a sensitivity of around $s=25 \mu \mathrm{K} \sqrt{\mathrm{sec}}$, are about a factor of two from the shot noise for the planned 14-month duration of the mission. Achieving greater sensitivity will necessitate some 
combination of larger or more detectors, broader frequency bands, and/or longer observation times.

CMB polarization may be used to address a number of cosmological issues aside from tensor perturbations. Polarization of order $5-10 \%$ of the temperature anisotropy is predicted in any model in which the CMB has a cosmological origin [30]. The absence of polarization or a polarization greatly in excess of that expected would force serious reconsideration of current cosmological models at the most fundamental level. Polarization can disentangle the peculiarvelocity contributions to the anisotropy on smaller angular scales [31]; only with a combined temperature-polarization map can a unique and unambiguous reconstruction of primordial density perturbations be made. Polarization can provide incontrovertible evidence for acoustic oscillations in the early Universe [32], help distinguish between competing cosmological models [33], constrain the ionization history of the Universe [20], probe cosmological magnetic fields [34], and improve cosmological-parameter determination [14]. The polarization of the CMB toward clusters can potentially be used to access other CMB surfaces of last scatter [35] or to learn about cluster physics [36,37].

Detection techniques and study of polarization foregrounds are in their infancy when compared with temperature anisotropies. However, we hope that the arguments presented here illustrate the fundamental importance of CMB polarization for physics and cosmology and motivate experimental developments in this direction.

\section{ACKNOWLEDGMENTS}

M.K. thanks George Smoot for useful conversations. This work was supported by D.O.E. contract DEFG02-92-ER 40699, NASA contract NAG5-3091, and the Alfred P. Sloan Foundation at Columbia, and by the Harvard Society of Fellows.
[1] A. H. Guth, Phys. Rev. D 23, 347 (1981).

[2] A. D. Linde, Phys. Lett. 108B, 389 (1982); A. Albrecht and P. J. Steinhardt, Phys. Rev. Lett. 48, 1220 (1982).

[3] J. M. Bardeen, P. J. Steinhardt, and M. S. Turner, Phys. Rev. D 28, 679 (1983); A. A. Starobinsky, Phys. Lett. 117B, 175 (1982); A. H. Guth and S.-Y. Pi, Phys. Rev. Lett. 49, 1110 (1982); S. W. Hawking, Phys. Lett. 115B, 295 (1982).

[4] M. Kamionkowski, D. N. Spergel, and N. Sugiyama, Astrophys. J. Lett. 426, L57 (1994).

[5] G. Jungman, M. Kamionkowski, A. Kosowsky, and D. N. Spergel, Phys. Rev. Lett. 76, 1007 (1996); Phys. Rev. D 54, 1332 (1996).

[6] J. R. Bond, G. Efstathiou, and M. Tegmark, astro-ph/9702100.

[7] See the MAP home page, URL http://map.gsfc.nasa.gov.

[8] See the Planck home page, URL http://astro.estec.esa.nl/SAgeneral/Projects/Cobras/cobras.html.

[9] L. F. Abbott and M. Wise, Nucl. Phys. B244, 541 (1984).

[10] A. R. Liddle and D. Lyth, Phys. Lett. B 291, 391 (1992); R. L. Davis et al., Phys. Rev. Lett. 69, 1856 (1992); M. S. Turner, Phys. Rev. D 48, 3502 (1993); F. Lucchin, S. Matarrese, and S. Mollerach, Astrophys. J. Lett. 401, L49 (1992); J. E. Lidsey and P. Coles, Mon. Not. R. Astron. Soc. 258, L57 (1992); F. C. Adams et al., Phys. Rev. D 47, 426 (1993). For a review, see J. Lidsey et al., Rev. Mod. Phys. 69, 373 (1997).

[11] M. Kamionkowski, A. Kosowsky, and A. Stebbins, Phys. Rev. Lett. 78, 2058 (1997).

[12] U. Seljak and M. Zaldarriaga, Phys. Rev. Lett. 78, 2054 (1997).

[13] R. G. Crittenden, D. Coulson, and N. G. Turok, Phys. Rev. D 52, 5402 (1995).

[14] M. Zaldarriaga, D. N. Spergel, and U. Seljak, astro-ph/9702157.

[15] M. S. Turner, Phys. Rev. D 48, 5539 (1992).

[16] L. Knox, Phys. Rev. D 52, 4307 (1995).
[17] L. A. Kofman and A. A. Starobinsky, Sov. Astron. Lett. 11, 271 (1986).

[18] M. Kamionkowski, A. Kosowsky, and A. Stebbins, Phys. Rev. D 55, 7368 (1997).

[19] M. Zaldarriaga and U. Seljak, Phys. Rev. D 55, 1830 (1997).

[20] M. Zaldarriaga, Phys. Rev. D 55, 1822 (1997).

[21] M. Tegmark, J. Silk, and A. Blanchard, Astrophys. J. 420, 484 (1994).

[22] Z. Haiman and A. Loeb, astro-ph/9611028 (1996).

[23] S. Dodelson, W. H. Kinney, and E. W. Kolb, Phys. Rev. D 56, 3207 (1997).

[24] D. H. Lyth, Phys. Rev. Lett. 78, 1861 (1997).

[25] A. Linde, Phys. Lett. 129B, 177 (1983).

[26] K. Freese, J. Frieman, and A. Olinto, Phys. Rev. Lett. 65, 3233 (1996).

[27] A. D. Linde, Phys. Lett. B 259, 38 (1991); Phys. Rev. D 49, 748 (1994); E. J. Copeland et al., ibid. 49, 6410 (1994); F. C. Adams and K. Freese, ibid. 41, 2449 (1990); G. D. Starkman, Report No. IASSNS-AST-90-37 (unpublished).

[28] L. Knox and M. S. Turner, Phys. Rev. Lett. 73, 3347 (1994).

[29] M. S. Turner, Phys. Rev. D 55, 435 (1997).

[30] M. Rees, Astrophys. J. Lett. 153, L1 (1968); J. Negroponte and J. Silk, Phys. Rev. Lett. 44, 1433 (1980); J. R. Bond and G. Efstathiou, Astrophys. J., Lett. Ed. 285, L47 (1984); Mon. Not. R. Astron. Soc. 226, 655 (1987); R. G. Crittenden et al., Astrophys. J. Lett. 417, L13 (1993).

[31] M. Zaldarriaga and D. Harari, Phys. Rev. D 52, 3276 (1995).

[32] A. Kosowsky (unpublished).

[33] U. Seljak, U.-L. Pen, and N. Turok, Phys. Rev. Lett. 79, 1615 (1997).

[34] A. Kosowsky and A. Loeb, Astrophys. J. 469, 1 (1996).

[35] M. Kamionkowski and A. Loeb, Phys. Rev. D 56, 4511 (1997).

[36] M. Gibilisco, astro-ph/9701203.

[37] A. Kosowsky and A. Loeb (in preparation). 